\title{
A Robust Fiber Bragg Grating Hydrogen Gas Sensor Using Platinum-Supported Silica Catalyst Film
}

\author{
Marina Kurohiji, ${ }^{1}$ Seiji Ichiriyama, ${ }^{2}$ Naoki Yamasaku, ${ }^{2}$ Shinji Okazaki $\left({ }^{\circ},{ }^{2}\right.$ Naoya Kasai, ${ }^{1}$ \\ Yusuke Maru, ${ }^{3}$ and Tadahito Mizutani ${ }^{4}$ \\ ${ }^{1}$ Graduate School of Environment and Information Sciences, Yokohama National University, 79-5 Tokiwadai, Hodogaya-ku, \\ Yokohama 240-8501, Japan \\ ${ }^{2}$ Graduate School of Engineering, Yokohama National University, 79-5 Tokiwadai, Hodogaya-ku, Yokohama 240-8501, Japan \\ ${ }^{3}$ Institute of Space and Astronautical Science, Japan Aero Space Exploration Agency, 3-1-1 Yoshinodai, Sagamihara 229-8510, Japan \\ ${ }^{4}$ Tsukuba Space Center, Japan Aerospace Exploration Agency, 2-1-1 Sengen, Tsukuba 305-8505, Japan
}

Correspondence should be addressed to Shinji Okazaki; okazaki-shinji-yp@ynu.ac.jp

Received 30 August 2017; Revised 22 November 2017; Accepted 11 December 2017; Published 15 February 2018

Academic Editor: Carlos Ruiz

Copyright ( 2018 Marina Kurohiji et al. This is an open access article distributed under the Creative Commons Attribution License, which permits unrestricted use, distribution, and reproduction in any medium, provided the original work is properly cited.

\begin{abstract}
A robust fiber Bragg grating (FBG) hydrogen gas sensor for reliable multipoint-leakage monitoring has been developed. The sensing mechanism is based on shifts of center wavelength of the reflection spectra due to temperature change caused by catalytic combustion heat. The sensitive film which consists of platinum-supported silica $\left(\mathrm{Pt} / \mathrm{SiO}_{2}\right)$ catalyst film was obtained using sol-gel method. The precursor solution was composed of hexachloroplatinic acid and commercially available silica precursor solution. The atom ratio of $\mathrm{Si}$ : Pt was fixed at 13:1. A small amount of this solution was dropped on the substrate and dried at room temperature. After that, the film was calcined at $500^{\circ} \mathrm{C}$ in air. These procedures were repeated and therefore thick hydrogen-sensitive films were obtained. The catalytic film obtained by 20 -time coating on quartz glass substrate showed a temperature change $75 \mathrm{~K}$ upon exposure to 3 vol. $\% \mathrm{H}_{2}$. For realizing robust sensor device, this catalytic film was deposited and FBG portion was directly fixed on titanium substrate. The sensor device showed good performances enough to detect hydrogen gas in the concentration range below lower explosion limit at room temperature. The enhancement of the sensitivity was attributed to not only catalytic combustion heat but also related thermal strain.
\end{abstract}

\section{Introduction}

Hydrogen is highly expected as an energy carrier in clean and sustainable energy systems which do not emit greenhouse gases because hydrogen gas is stable under normal storage conditions and produces only water theoretically in power generation processes. Although hydrogen should be produced using renewable natural energy sources, suitable areas where these sources can be easily and efficiently utilized are limited to specific remote regions. Therefore, various hydrogen storage and transportation facilities such as the ultrahigh-pressure tank or the long pipeline would be required in order to realize such energy systems [1-3].

Hydrogen exhibits many hazardous characteristics such as wide flammable range, small ignition energy, and ease of leakage due to the small molecular size. If hydrogen leaks from large facilities associated with production, transportation, and storage, it could lead to serious explosion accidents. For ensuring safety of the energy systems, it is important to detect the leakage of hydrogen gas as fast as possible using a sensitive gas sensor since it is colorless and odorless gas $[4,5]$. However, a large number of sensor devices would be required for monitoring the leakage over a wide area where various huge energy facilities including pipeline are operating. Although semiconductor-type and electrochemical gas sensors are widely used for hydrogen sensing [6], these sensor devices could not satisfy needs for distributed or quasidistributed (multipoint) leakage detection due to spot-type (single-point) characteristics. This is attributed to the energy consumption for device heating to improve their sensing 


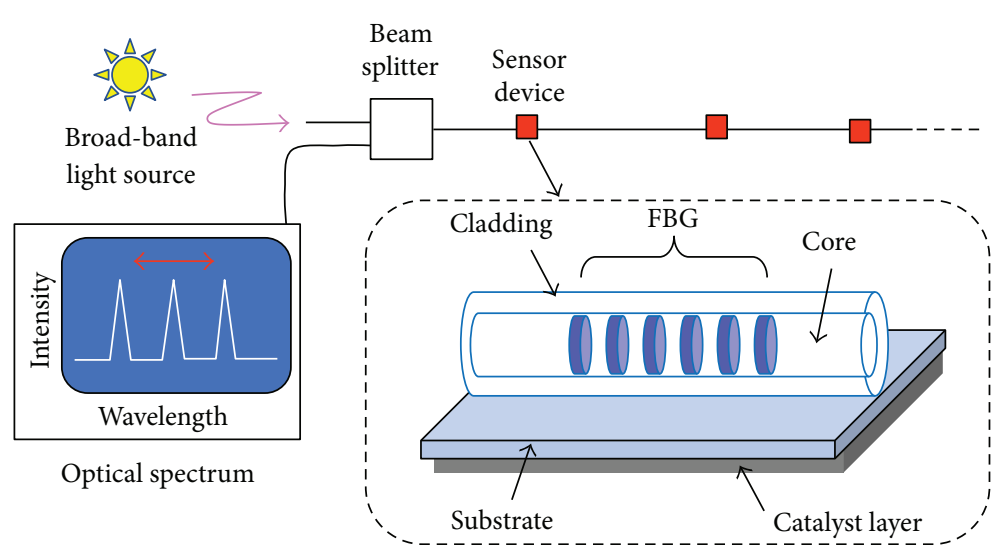

FIGURE 1: Operation principle of multipoint fiber Bragg grating sensor system and structure of catalyst-type hydrogen gas sensor device.

performance. In addition, the sensor device which has the electrode structure might accidentally cause static electrostatic discharge or spark. In the worst-case scenario, there is a risk that the sensor device may become an ignition source in explosive conditions [7].

Fiber-optic methods have a potential for a distributed leakage monitoring [8].

The leakage points along a fiber cable could be determined, and three-dimensional spatial measurement by winding the fiber-optic sensor cable around the facilities would be easily achieved. Furthermore, significantly valuable characteristics such as explosion proof and immunity to electromagnetic noise are noteworthy for practical applications. Many types of optical fiber hydrogen sensors have been proposed, including evanescent wave absorption devices $[9,10]$, micromirror optode [11, 12], Mach-Zehnder interferometers [13], surface plasmon resonance (SPR) devices [14], and fiber Bragg gratings (FBG) [15-17]. Among them, the fiber-optic sensor based on evanescent wave absorption is able to detect the leakage point by time-domain reflectometry using light pulse technique [18]. However, propagation loss of the sensor device and dynamic range of OTDR instruments limit the sensing length of the fiber-optic sensor device. On the other hand, a multipoint sensor system is easily realized on a single fiber cable and can also identify leakage points by using FBG sensor through monitoring Bragg wavelength [19]. It is well known that Pd or Pd-based alloy expands by hydrogen absorption and this phenomenon has been already utilized in various FBG-type hydrogen sensors [20-22]. However, FBG device structures needed to be modified by chemical etching or mechanical polishing for enhancing the sensitivity. In recent years, it was found that a highly sensitive FBG sensor was achieved by utilizing temperature change induced by exothermic oxidation reaction of hydrogen on $\mathrm{Pt} / \mathrm{WO}_{3}$ catalyst. This sensor structure is very simple and robust. The fabrication process is also very easy [16, 23]. The catalytic activity of noble metal-loaded oxide is considerably influenced by its structure, particle size, supporting materials, and so forth. The authors have recently assessed the catalytic activities of various oxide-supported platinum particles prepared by a sol-gel method. As a result, a highly sensitive FBG-type sensor using $\mathrm{Pt} / \mathrm{SiO}_{2}$ film has been developed [24].
In this work, this catalytic film was deposited by the sol-gel method and an FBG portion was directly fixed on a titanium substrate in order to realize a robust device for multipoint sensor system. The film was characterized by optical microscope, XRD, and EPMA, and the sensing performances of this sensor structure were evaluated.

\section{Sensing Principle}

An FBG was developed as a kind of optical passive filter, which has a periodic structure of the refractive index in the fiber core region. Figure 1 shows the schematic diagram of multipoint FBG sensor system and the structure of catalysttype gas sensor device. If broadband light enters an optical fiber cable with multiple FBG devices which have different Bragg wavelengths, the FBG sensor system reflects their particular wavelength light expressed by the following equation:

$$
\lambda_{\mathrm{B}}=2 n d,
$$

where $\lambda_{\mathrm{B}}[\mathrm{nm}]$ is called Bragg wavelength, $n[-]$ is the effective refractive index of the core region, and $d[\mathrm{~nm}]$ is the grating period [25].

It is well known that Bragg wavelength is sensitive to change in the grating period or effective refractive index of the core region caused by a change in strain or temperature. This mechanism is extensively applied to quasi-distributed strain or temperature sensing. Furthermore, the feature is frequently utilized as a primary transduction element for various type of sensors. For example, FBG hydrogen gas sensor can be easily developed by a combination of suitable device structure and hydrogen-sensitive material which can convert the information about hydrogen concentration into a strain or temperature change.

In this study, the FBG sensor based on a temperature change generated by catalytic reaction heat of hydrogen gas was fabricated. In order to realize this type of sensor device, a platinum-supported silica film was used as a hydrogensensitive material, which was coated on a durable substrate and then an FBG device was fixed on this, as shown in Figure 1. In the presence of hydrogen gas in the air, the following reaction readily proceeds on platinum catalysts even at room temperature. 


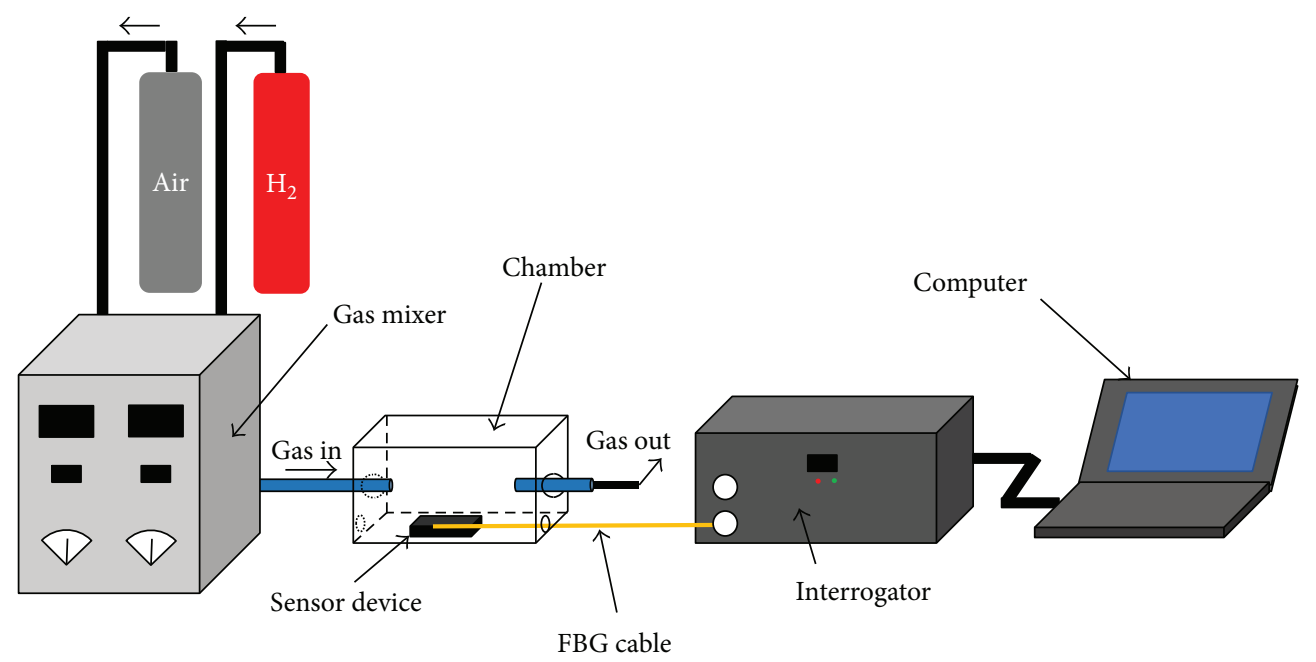

FIGURE 2: Structure of a sensor device and experimental setups used for characterizing sensor response.

$$
\mathrm{H}_{2}+\frac{1}{2} \mathrm{O}_{2} \stackrel{\mathrm{Pt}}{\rightarrow} \mathrm{H}_{2} \mathrm{O}
$$

The large and exothermic combustion heat $(-245 \mathrm{~kJ} /$ $\mathrm{mol}$ ) is released from the catalyst layer. [26]. It would cause a temperature change of FBG device corresponding to hydrogen concentration level. By using an interrogation system for monitoring Bragg wavelength shift, it would be possible to detect multipoint leakage of hydrogen gas.

\section{Experimental}

3.1. Preparation of the Catalyst Layer on Quartz Glass and Titanium Substrates. A hydrogen-sensitive material was prepared by the sol-gel method. A precursor solution of silicon dioxide ( $\mathrm{Si}-05 \mathrm{~S}\left(\mathrm{SiO}_{2}\right.$ about 5 wt.\%); Kojundo Chemical Laboratory Co.) was used to form the support. A hexachloroplatinic acid solution $\left(1.9 \times 10^{-3} \mathrm{M}\right)$ of $170 \mu \mathrm{L}$ was mixed with the precursor solution of $5 \mathrm{~mL}$ so that the atom ratio between Si and Pt became 13:1. The catalyst layer was deposited on substrates of quartz glass and titanium (purity $>99 \%$ ). Dimensions of glass and Ti substrates were $18 \times 18 \times 0.15$ and $20 \times 20 \times 1.5 \mathrm{~mm}$, respectively. The catalyst precursor of $30 \mu \mathrm{L}$ at a time was dropped on the substrate by a micropipette. After drying, these films were subsequently calcined at $500^{\circ} \mathrm{C}$ in the air for 60 minutes. After calcination process, the furnace was naturally cooled down by turning off electricity. In order to enhance the sensitivity, this process was repeated over and over again.

Optical microscope (VH-Z500R (Keyence Co.)) and scanning electron microscope (SEM VE-8800 (Keyence Co.)) were used to observe the morphology of the obtained samples. The crystal structures of catalytic film were investigated by X-ray diffraction (XRD SmartLab (Rigaku Co.)) in a continuous mode with $\mathrm{Cu} \mathrm{K} \alpha$ radiation $(\lambda=1.54059 \AA)$. The scanning was ranging from 10 to 90 degrees.

For evaluating the surface and the cross-sectional conditions of the catalyst layer, electron probe micro analysis (EPMA) was conducted with JXA-8530F (JEOL Co.). The electron acceleration was $15 \mathrm{kV}$; the diameter of backscatter electronic beam was set to $100 \mu \mathrm{m}$.

3.2. Structure of Sensor Device and Evaluation of Performance. Hydrogen detection experiments were conducted by the FBG with the catalyst layer.

Figure 2 shows the experimental setup. The FBG temperature sensor was attached on the surface of the catalyst layer with adhesive tape in order to measure temperature of the film. In the case of fabricating the sensor device using the titanium substrate, the bare FBG device was directly immobilized on the opposite side of the catalyst layer with cyanoacrylate adhesive. The central wavelength of the FBG device was monitored by a Laser Swept Interrogator (sm125, Micron Optics Inc.) at a sampling frequency of $2 \mathrm{~Hz}$ and a wavelength resolution of $1 \mathrm{pm}$.

Dry air was used as carrier gas and mixed with pure hydrogen through a gas mixing device (GB-2C, Kofloc), and the flow rate was monitored and regulated at $1.0 \mathrm{~L} / \mathrm{min}$. The different concentrations of hydrogen gas from 0 to $4.0 \mathrm{vol}$ \% were fed to a home-made gas chamber whose volume is about $48 \mathrm{~mL}$. All experiments for the characterization of sensor response were conducted at room temperature.

\section{Results and Discussion}

4.1. Characterizations of the Catalyst Film and Its Ability for Hydrogen Detection. Figure 3 shows the temperature variations of glass substrates immobilizing $\mathrm{Pt}-\mathrm{SiO}_{2}$ catalyst layers with time when the devices are exposed to 2 vol.\% hydrogencontaining air. In the case of the device coated with catalyst layer 5 times, the temperature gradually increased and the temperature variation after $180 \mathrm{~s}$ was about $2 \mathrm{~K}$. It indicates that the catalytic combustion heat is easily captured by this simple configuration of the device. After replacing the test gas to the air, the temperature was readily recovered by cooling mainly based on Newton's law. The remarkable temperature variation of about $15 \mathrm{~K}$ was observed with exposure to the 2 vol.\% hydrogen-containing air for the device coated with catalyst layer 15 times. While the amount of catalyst 


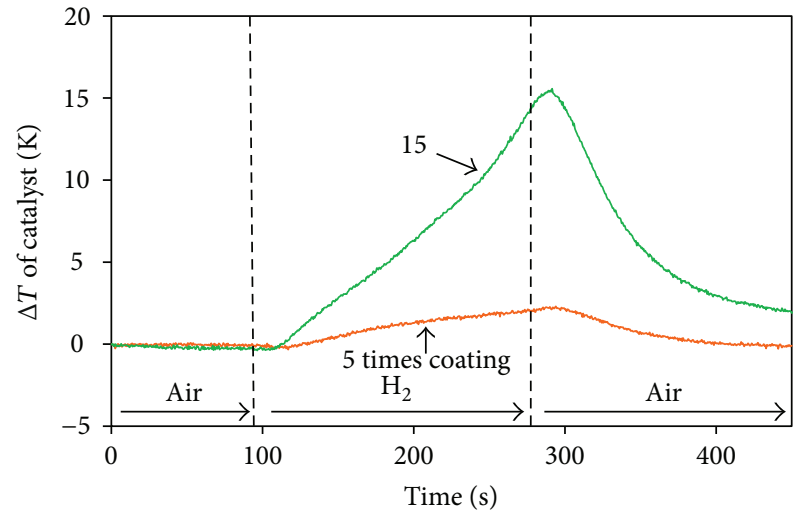

FIgURE 3: Temperature variations of glass substrates immobilizing $\mathrm{Pt}-\mathrm{SiO}_{2}$ catalyst layers (5 times coating) with time upon exposure to 2 vol.\% hydrogen-containing air.

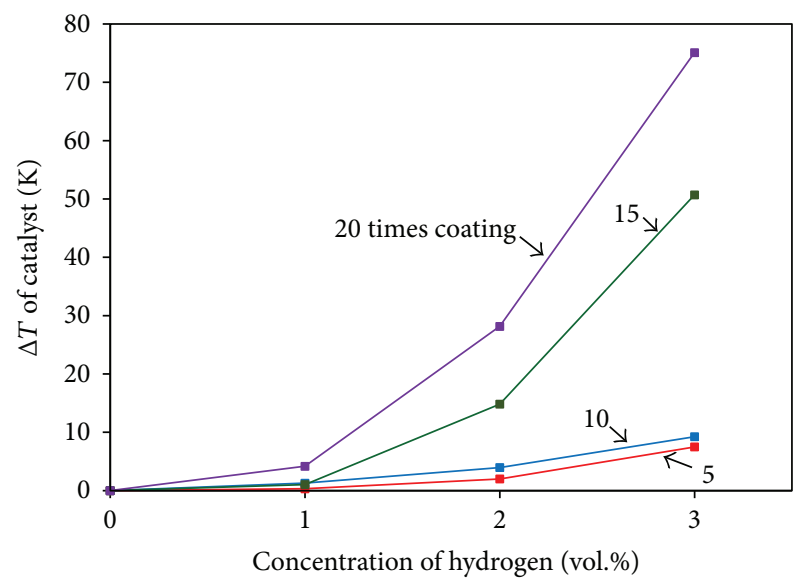

FIGURE 4: Relationship between temperature changes of the glass substrate at different numbers of the repeated catalyst coating and hydrogen concentration.

tripled, the sensitivity was considerably enhanced more than expected. The active surface area of catalyst would be effectively increased by repeated coating. However, the slight autocatalytic temperature increase was observed over the temperature variation of $10 \mathrm{~K}$. It suggests that the rate constant of the catalytic combustion reaction could not be considered as a constant value because the increase in this rate constant based on the Arrhenius' equation would not be negligibly small beyond this temperature range. Therefore, it should be also recognized that there is a risk of localized thermal runaway if the hydrogen concentration and the sensitivity of the sensor device are too high. Due to this trade-off relation between the sensitivity and the potential risk of the device itself, the optimization and the safety assessment of sensor structure would be required for practical applications.

Figure 4 shows the relationship between temperature change of the glass substrate at different numbers of the repeated catalyst coating and hydrogen concentration. The calibration curves were not linear, and the sensitivity tended to increase in the higher concentration range. Furthermore, it is found that the sensitivity could be enhanced by increasing the number of repeated coating although the sensitivities of 5

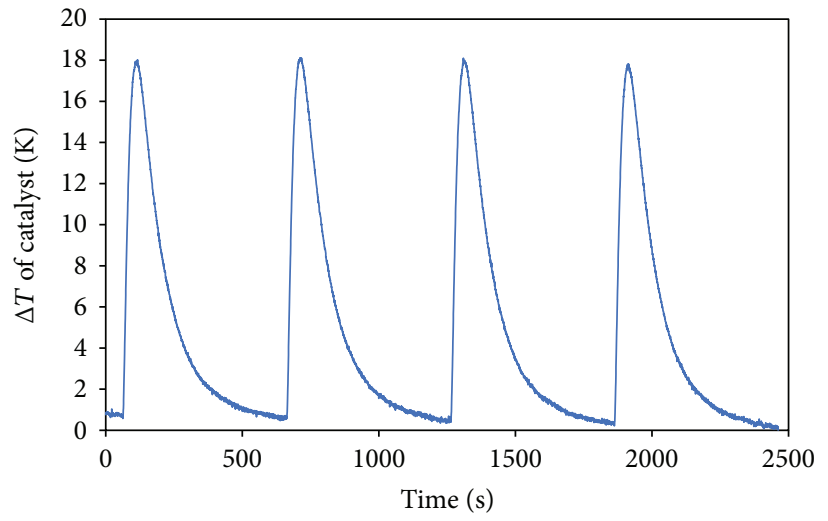

FIgURE 5: Repeatability in thermal response of the glass substrates immobilizing $\mathrm{Pt}-\mathrm{SiO}_{2}$ catalyst layers (20 times) to 2 vol.\% hydrogen-containing air.

and 10 times catalyst coating were almost same. Therefore, most of the embedded catalysts would preserve its activity even if they exit at a deep position of catalytic layer near the substrate surface. It suggests that the sensitivity could be easily controlled by this fabrication method. In our experimental condition, the repeated coating was succeeded up to 20 times without peeling and detachment of catalyst layer and some kind of additives such as polymer binders would be needed for additional repeated coating.

In order to check the hysteresis behaviors, the repeated response characteristic of the glass substrate immobilizing the $\mathrm{Pt}-\mathrm{SiO}_{2}$ layers (20 times) to the 2 vol.\% hydrogencontaining air was evaluated. As shown in Figure 5, this catalytic film has good repeatability and there is slight hysteresis behavior in a short period of measurement.

Figure 6 represents the microscopic photographs and the SEM pictures of the surface of catalyst film. It is found that many cracks were formed, and they made the catalyst layer become an aggregation of tiny flake-like particles. Widths of the cracks and diameters of the particles tended to reduce with increasing the number of repeated coating. Furthermore, the SEM pictures (Figures 6(c) and 6(d)) indicate that the structure of the catalyst film inside the crack would be very complex and they allow hydrogen gas to penetrate throughout the film.

Figure 7 gives the XRD pattern of the catalyst film on the glass substrate (repeated catalyst coating: 20 times). The XRD diffraction peaks were matched with those of XRD standard cards of Pt (ICDD PDF-2, number 01-071-6560) and $\mathrm{SiO}_{2}$ (ICDD PDF-2, number 01-082-1554). Low intensity and broadness of the peak around $2 \theta$ values of 22.15 indicate that the crystallographic structure of $\mathrm{SiO}_{2}$ as a catalyst support is amorphous or very small nano-crystalline. On the other hand, sharp Pt peaks are observed at $2 \theta$ values of $39.77,46.25,67.47,81.27$, and 85.72 . They indicate that Pt exists as metallic crystals in the catalyst film. The average crystallite size of about $4 \mathrm{~nm}$ was determined by Scherer's formula.

The distribution of such tiny catalyst particle throughout the film was investigated. Figures 8(a) and 8(b) show EPMA images of the catalyst film with respect to the surface and the 


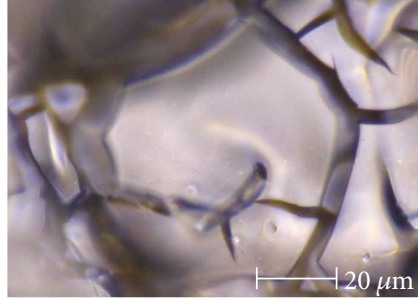

Catalyst coating: 5 times

(a)

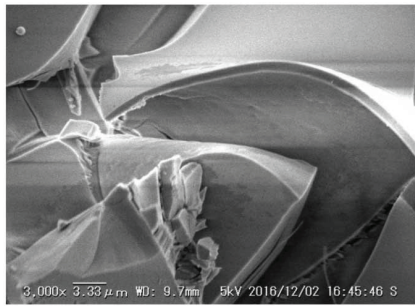

5 times

(c)

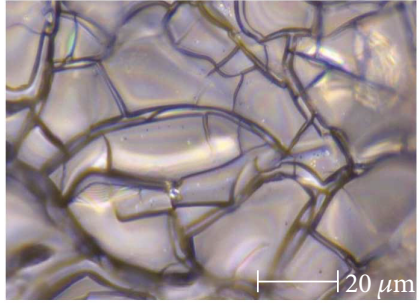

20 times

(b)

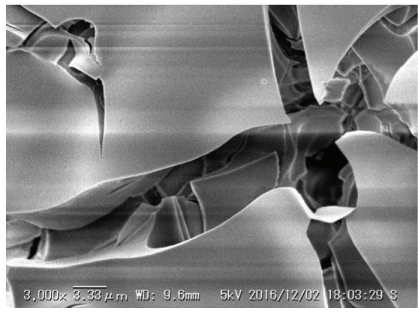

20 times

(d)

Figure 6: Optical micrographs $(\mathrm{a}, \mathrm{b})$ and SEM pictures $(\mathrm{c}, \mathrm{d})$ of the surface of catalyst film whose numbers of repeated coating are 5 and 20 times, respectively.

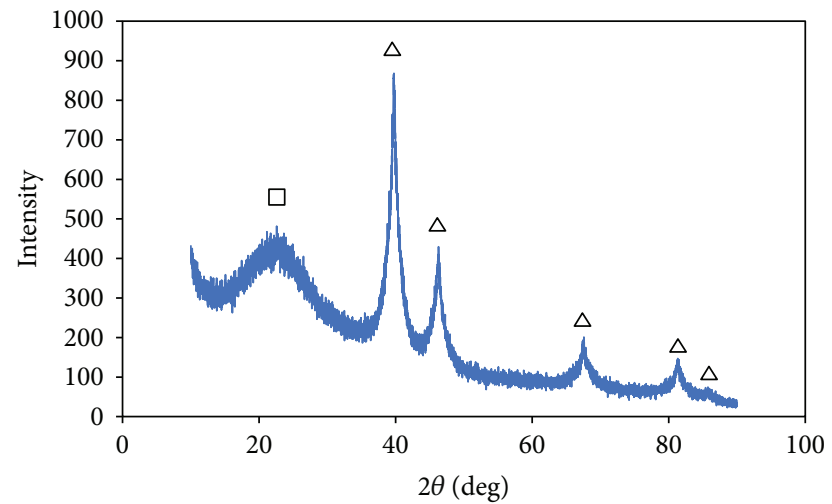

$\triangle \mathrm{Pt}$

$\square \mathrm{SiO}_{2}(\mathrm{NCL}-1)$

FIGURE 7: XRD pattern of the catalyst film on glass substrate (repeated catalyst coating: 15 times).

cross section, respectively. These images represent that there is no remarkable segregation of $\mathrm{Pt}$ and all elements are relatively well dispersed. However, it is found that Pt catalysts seem to be concentrated at the boundary between the film and the glass substrate as shown in the cross-sectional image. Since the film has a lot of cracks and hollows, the catalyst precursor solution easily permeates the film. Therefore, the content of Pt near the boundary would be slightly higher than that at the surface. Considering that the sensitivity increased with the number of repeated coating, the mass transfer of hydrogen and oxygen molecules would not be inhibited and a reduction of reaction surface area of Pt catalysts by agglomeration would not occur. When catalytic combustion occurs, water is produced. This water stays in cracks and hollows and may prevent hydrogen from reaching inside of the catalyst.
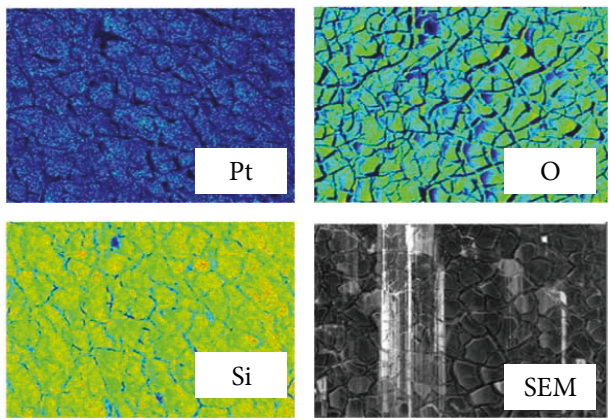

$\longmapsto 50 \mu \mathrm{m}$

(a)
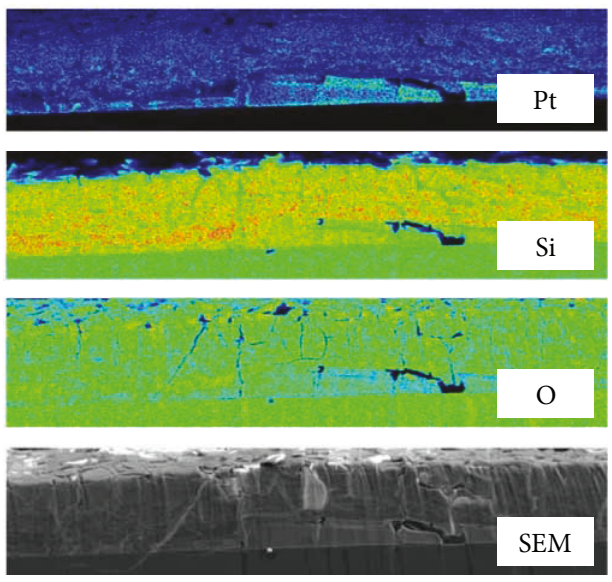

$\longmapsto 50 \mu \mathrm{m}$

(b)

FIGURE 8: Element mapping (EPMA images) of the catalyst film on glass substrate (repeated catalyst coating: 20 times) with respect to the surface (a) and the cross section (b), respectively. 


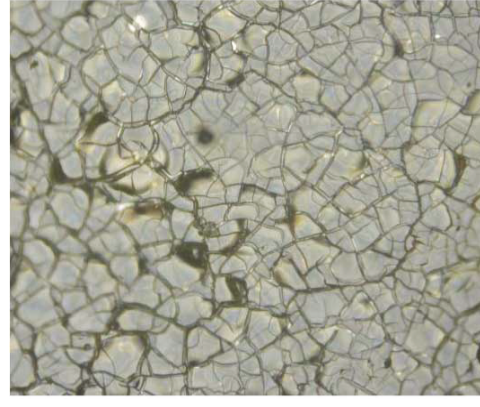

$\longmapsto 50 \mu \mathrm{m}$

(a)

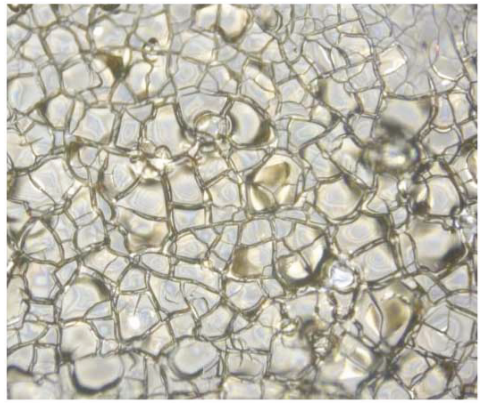

$\longmapsto 50 \mu \mathrm{m}$

(b)

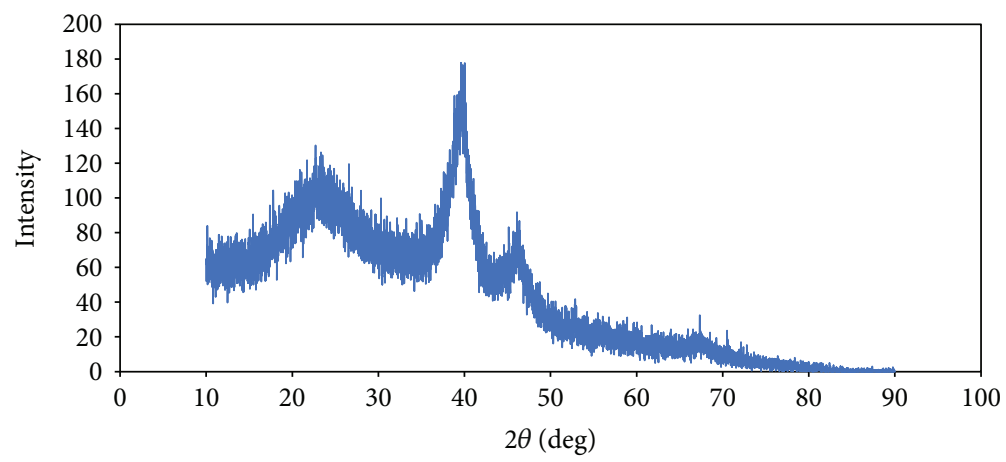

(c)

Figure 9: Comparison of microscopic photographs of the film surface immobilized on Ti substrate (a) with that on glass substrate (b) and XRD pattern of catalyst film on the Ti substrate (c) (repeated catalyst coating: 20 times).

Then the produced heat can be decreased because hydrogen cannot contact with Pt. Excess sinter also seems to deteriorate the performance of the catalyst layers because it may cause disappearance of hollows.

4.2. Hydrogen Detection Using the Catalyst Film on the Ti Substrate. In order to realize a robust and sensitive sensor device, the same catalyst film (repeated catalyst coating: 20 times) was tried to be immobilized on the Ti substrate and sensor performances were evaluated. Figure 9 shows the comparison of microscopic photographs of the film surface immobilized on the Ti substrate with that on the glass substrate. It is clear that there is almost no difference in appearance. Figure 9 gives the XRD pattern of the catalyst film on the Ti substrate (repeated catalyst coating: 20 times). The XRD diffraction peaks were matched with those of XRD standard cards of Pt (ICDD PDF-2, number 01-071-6560) and $\mathrm{SiO}_{2}$ (ICDD PDF-2, number 01-082-1554). There is almost no difference in $\mathrm{Pt}$ and $\mathrm{SiO}_{2}$ peaks obtained from the glass and Ti substrates.

Figure 10 shows the temperature variations of the Ti substrate immobilizing $\mathrm{Pt}-\mathrm{SiO}_{2}$ catalyst layers with time when the device is exposed to the 2 vol.\% hydrogen-containing air. The temperature gradually increased, and the temperature variation after the exposure for $200 \mathrm{~s}$ was about $2 \mathrm{~K}$. Compared with results using glass substrates, this temperature variation is significantly decreased. Although a numerical simulation, such as finite element analysis which is based on the thermal model consisting of an FBG device, catalyst

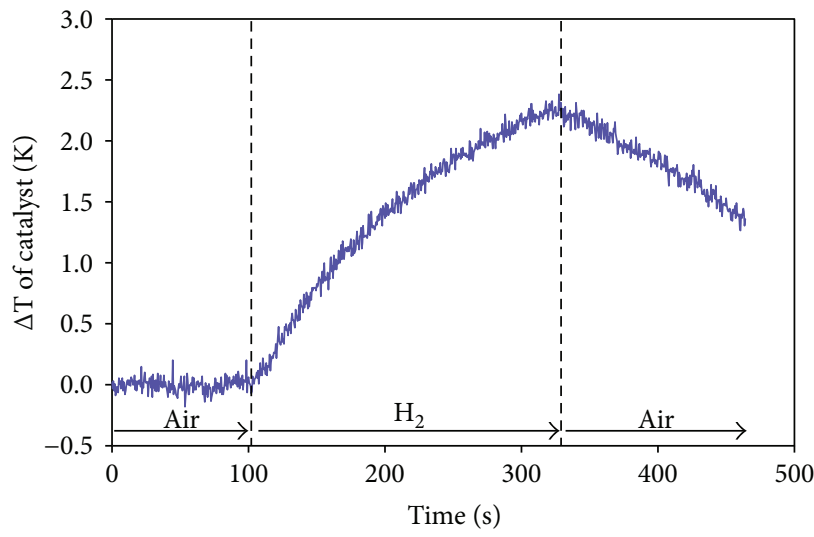

Figure 10: Temperature variations of Ti substrates immobilizing $\mathrm{Pt}-\mathrm{SiO} 2$ catalyst layers with time when the devices are exposed to 2 vol.\% hydrogen-containing air.

film, and a substrate, would be required for more detailed quantitative analysis and optimization of sensor performance, this result suggests that produced catalytic heat easily dissipated outside since the heat-transfer coefficient of the $\mathrm{Ti}$ substrate is much larger than that of glass.

As a next step, a bare FBG device was immobilized on the Ti substrate and sensor performances were evaluated. Figure 11 shows the typical sensor response curves at different concentrations of hydrogen gas in the air. It is demonstrated that this sensor device can detect hydrogen gas in the concentration range below lower explosion limit. 


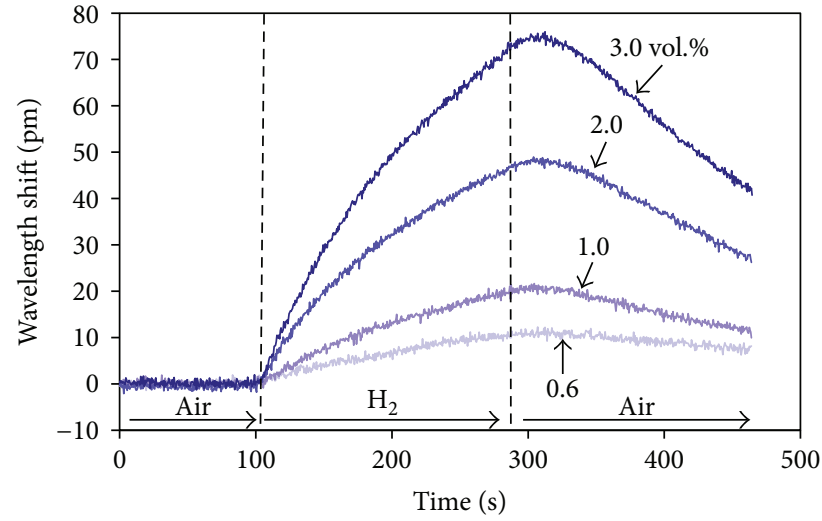

FIGURE 11: Typical response curves of the sensor where a bare FBG device was directly immobilized on the Ti substrate at different concentrations of hydrogen gas in air.

The wavelength shift of $48 \mathrm{pm}$ was observed with exposure to 2 vol.\% hydrogen gas. Generally, the Bragg wavelength of a typical FBG device linearly shifts with both temperature and strain, and the temperature and strain sensitivities are about $10 \mathrm{pm} /{ }^{\circ} \mathrm{C}$ and $1 \mathrm{pm} / \mu \varepsilon$, respectively. In this case, the temperature rise of $2 \mathrm{~K}$ as shown in Figure 10 would cause $20 \mathrm{pm}$ shift. However, the sensitivity is approximately twice as high as that estimated by only temperature variation. It suggests that the enhancement of sensitivity would result from thermal strain which is caused by the difference in the thermal expansion coefficient between the Ti substrate and the FBG device mainly made of quartz glass. The linear expansion coefficient of $\mathrm{Ti}$ is larger than that of quartz glass. The values are $8.6 \times 10^{-6} \mathrm{~K}^{-1}$ for Ti and $0.55 \times 10^{-6} \mathrm{~K}^{-1}$ for quartz glass. Then, it is assumed that thermal strain is dominated by that of $\mathrm{Ti}$ substrate since the thickness of the $\mathrm{Ti}$ substrate $(1.5 \mathrm{~mm})$ is much larger than the diameter of the FBG device $(0.125 \mathrm{~mm})$. The thermal strain $\varepsilon[-]$ at the FBG portion can be roughly calculated by the linear expansion coefficient $\alpha$ $\left[\mathrm{K}^{-1}\right]$ of Ti and temperature change $\Delta[\mathrm{K}]$ as follows:

$$
\varepsilon=\alpha \Delta T .
$$

In this case, additional Bragg wavelength shift of about $16 \mathrm{pm}$ was estimated. Therefore, it can be considered that the sensitivity is enhanced by not only temperature rise but also thermal strain. Similar enhancement on the sensitivity of an FBG-type device whose periphery a Ni metal thick layer was deposited on has already been reported [27].

Figure 12 represents the calibration curve between the shift in Bragg wavelength and hydrogen concentration. A good linearity which would correspond to first order reaction kinetics was observed. In our experimental condition, peakto-peak baseline noise signal was about $2 \mathrm{pm}$. Calculating the detection limit equal to signal-to-noise ratio of 3 , the value of $0.2 \mathrm{vol} . \%$ was obtained. Although the sensitivity of this sensor is enough for the application to multipointleakage monitoring, the response rate is relatively slow as shown in Figure 11.

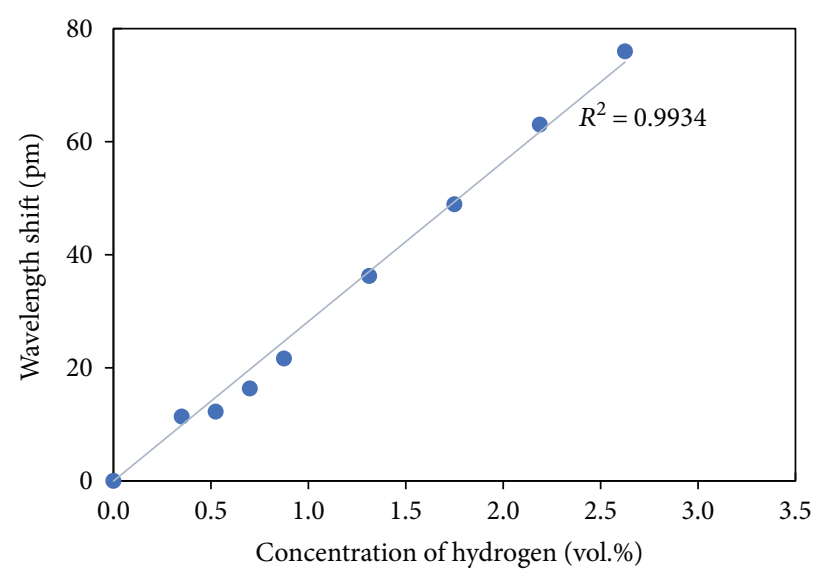

FIgURE 12: Calibration curve between shift in Bragg wavelength and hydrogen concentration.

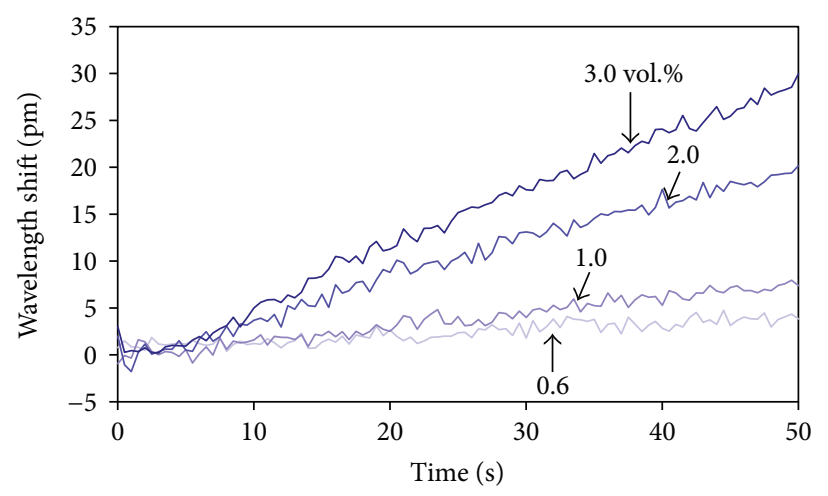

FIGURE 13: Initial response behavior after exposure to hydrogencontaining air.

Figure 13 shows the initial response behavior after exposure to hydrogen-containing air. Considering the detection limit equal to signal-to-noise ratio of 3 and resolution of the FBG interrogator is less than $1 \mathrm{pm}$, hydrogen leakage at a concentration level of 1.0 vol.\% could be detected within $30 \mathrm{~s}$ if the differential signal would also be exploited.

\section{Conclusion}

$\mathrm{Pt} / \mathrm{SiO}_{2}$ catalyst layers were successfully deposited on both quartz glass and titanium substrates by sol-gel method. XRD and EPMA results showed that the catalyst film had a lot of cracks. It indicates that hydrogen gas easily enters and diffuses into the film. Therefore, Pt particles uniformly dispersed in the film would be efficiently utilized for the catalytic combustion reaction. The FBG sensor devices using the catalyst film repeated coating of precursor solution 20 times on a titanium substrate were able to detect lowconcentration hydrogen gas sensitively. Furthermore, it was clarified that the sensitivity was enhanced due to the effect of thermal strain caused by a mismatch in the thermal expansion coefficient between the titanium substrate and the FBG device made of quartz glass. Considering practical applications, this robust sensor structure is suitable for its handling and mounting. The as-needed sensitivity can be easily 
designed and achieved by the number of catalyst coating. It would also prevent a potential risk of ignition upon exposure to high concentration of hydrogen gas. Further studies on selectivity and long-term stability of catalyst film would be required for the next step and reported in due course.

\section{Conflicts of Interest}

The authors declare that there is no conflict of interests regarding the publication of this paper.

\section{References}

[1] S. Dunn, "Hydrogen futures: toward a sustainable energy system," International Journal of Hydrogen Energy, vol. 27, no. 3, pp. 235-264, 2002.

[2] M. Momirlana and T. N. Veziroglu, "The properties of hydrogen as fuel tomorrow in sustainable energy system for a cleaner planet," International Journal of Hydrogen Energy, vol. 30, no. 7, pp. 795-802, 2005.

[3] S. Dutta, "A review on production, storage of hydrogen and its utilization as an energy resource," Journal of Industrial and Engineering Chemistry, vol. 20, no. 4, pp. 1148-1156, 2014.

[4] V. A. Petukhov, I. M. Naboko, and V. E. Fortov, "Explosion hazard of hydrogen-air mixtures in the large volumes," International Journal of Hydrogen Energy, vol. 34, no. 14, pp. 59245931, 2009.

[5] D. A. Crowl and Y.-D. Jo, "The hazards and risks of hydrogen," Journal of Loss Prevention in the Process Industries, vol. 20, no. 2, pp. 158-164, 2007.

[6] T. Hübert, L. Boon-Brett, G. Black, and U. Banach, "Hydrogen sensors - a review," Sensors and Actuators B: Chemical, vol. 157, no. 2, pp. 329-352, 2011.

[7] G. R. Astbury and S. J. Hawksworth, "Spontaneous ignition of hydrogen leaks: a review of postulated mechanisms," International Journal of Hydrogen Energy, vol. 32, no. 13, pp. 21782185, 2007.

[8] K. Bremer, M. Meinhardt-Wollweber, T. Thiel et al., "Sewerage tunnel leakage detection using a fibre optic moisturedetecting sensor system," Sensors and Actuators A: Physical, vol. 220, pp. 62-68.

[9] K. Schroeder, W. Ecke, and R. Willsch, "Optical fiber Bragg grating hydrogen sensor based on evanescent-field interaction with palladium thin-film transducer," Optics and Lasers in Engineering, vol. 47, no. 10, pp. 1018-1022, 2009.

[10] S. Sekimoto, H. Nakagawa, S. Okazaki et al., "A fiber-optic evanescent-wave hydrogen gas sensor using palladiumsupported tungsten oxide," Sensors and Actuators B: Chemical, vol. 66, no. 1-3, pp. 142-145, 2000.

[11] M. A. Butler, "Micromirror optical-fiber hydrogen sensor," Sensors and Actuators B: Chemical, vol. 22, no. 2, pp. 155163, 1994.

[12] X. Be'venot, A. Trouillet, C. Veillas, H. Gagnaire, and M. Cle'ment, "Hydrogen leak detection using an optical fibre sensor for aerospace applications," Sensors and Actuators B: Chemical, vol. 67, no. 1-2, pp. 57-67, 2000.

[13] M. Wang, D. N. Wang, M. Yang, J. Cheng, and J. Li, "In-line Mach-Zehnder interferometer and FBG with Pd film for simultaneous hydrogen and temperature detection," Sensors and Actuators B: Chemical, vol. 202, pp. 893-896, 2014.
[14] X. Wang, Y. Tang, C. Zhou, and B. Liao, "Design and optimization of the optical fiber surface plasmon resonance hydrogen sensor based on wavelength modulation," Optics Communications, vol. 298-299, pp. 88-94, 2013.

[15] J. Dai, M. Yang, Z. Yang et al., "Enhanced sensitivity of fiber Bragg grating hydrogen sensor using flexible substrate," Sensors and Actuators B: Chemical, vol. 196, pp. 604-609, 2014.

[16] J. Dai, M. Yang, Z. Yang et al., "Performance of fiber Bragg grating hydrogen sensor coated with Pt-loaded $\mathrm{WO}_{3}$ coating," Sensors and Actuators B: Chemical, vol. 190, pp. 657-663, 2014.

[17] J. Dai, M. Yang, X. Yu, K. Cao, and J. Liao, "Greatly etched fiber Bragg grating hydrogen sensor with $\mathrm{Pd} / \mathrm{Ni}$ composite film as sensing material," Sensors and Actuators B: Chemical, vol. 174, pp. 253-257, 2012.

[18] J. Buerck, S. Roth, K. Kraemer, and H. Mathieu, "OTDR fiberoptical chemical sensor system for detection and location of hydrocarbon leakage," Journal of Hazardous Materials, vol. 102, no. 1, pp. 13-28, 2003.

[19] J. Dakin and B. Culshaw, Optical Fiber Sensors, vol. 4, Artech House publishers, Boston, UK, 1997.

[20] G.-m. Ma, C.-r. Li, Y.-t. Luo, M. Rui-duo, and L. Wang, "High sensitive and reliable fiber Bragg grating hydrogen sensor for fault detection of power transformer," Sensors and Actuators B: Chemical, vol. 169, pp. 195-198, 2012.

[21] X. Zhou, Y. Dai, M. Zou, J. M. Karanja, and M. Yang, "FBG hydrogen sensor based on spiral microstructure ablated by femtosecond laser," Sensors and Actuators B: Chemical, vol. 236, pp. 392-398, 2016.

[22] J. Dai, M. Yang, X. Yu, and H. Lu, "Optical hydrogen sensor based on etched fiber Bragg grating sputtered with $\mathrm{Pd} / \mathrm{Ag}$ composite film," Optical Fiber Technology, vol. 19, no. 1, pp. 26-30, 2013.

[23] M. Yang, Z. Yang, J. Dai, and D. Zhang, "Fiber optic hydrogen sensors with sol-gel $\mathrm{WO}_{3}$ coatings," Sensors and Actuators B: Chemical, vol. 166-167, pp. 632-636, 2012.

[24] S. Masuzawa, S. Okazaki, Y. Maru, and T. Mizutani, "Catalysttype-an optical fiber sensor for hydrogen leakage based on fiber Bragg gratings," Sensors and Actuators B: Chemical, vol. 217, pp. 151-157, 2015.

[25] T. Erdogan, "Fiber Grating Spectra," Journal of Lightwave Technology, vol. 15, no. 8, pp. 1277-1294, 1997.

[26] C. Appel, J. Mantzaras, R. Schaeren, R. Bombach, and A. Inauen, "Catalytic combustion of hydrogen-air mixtures over platinum: validation of hetero/homogeneous chemical reaction schemes," Clean Air: International Journal on Energy for a Clean Environment, vol. 5, no. 1, pp. 21-44, 2004.

[27] Y. Li, Z. Hua, F. Yan, and P. Gang, "Metal coating of fiber Bragg grating and the temperature sensing character after metallization," Optical Fiber Technology, vol. 15, no. 4, pp. 391-397, 2009. 


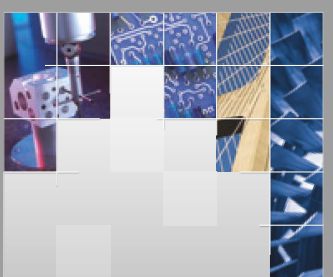

\section{Enfincering}
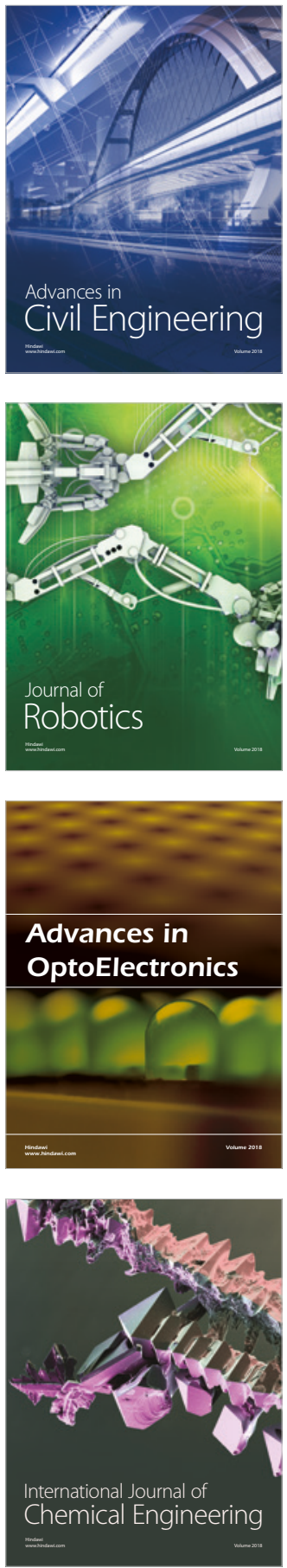

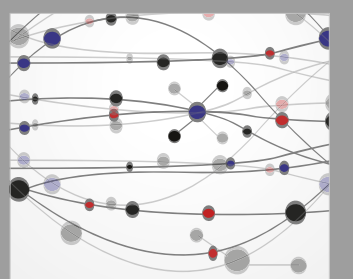

\section{Rotating \\ Machinery}

The Scientific World Journal

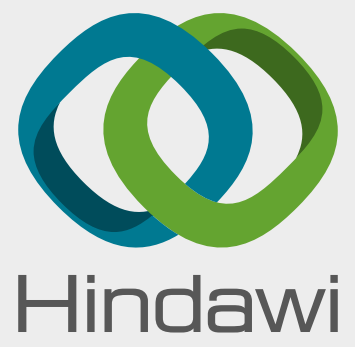

Submit your manuscripts at

www.hindawi.com
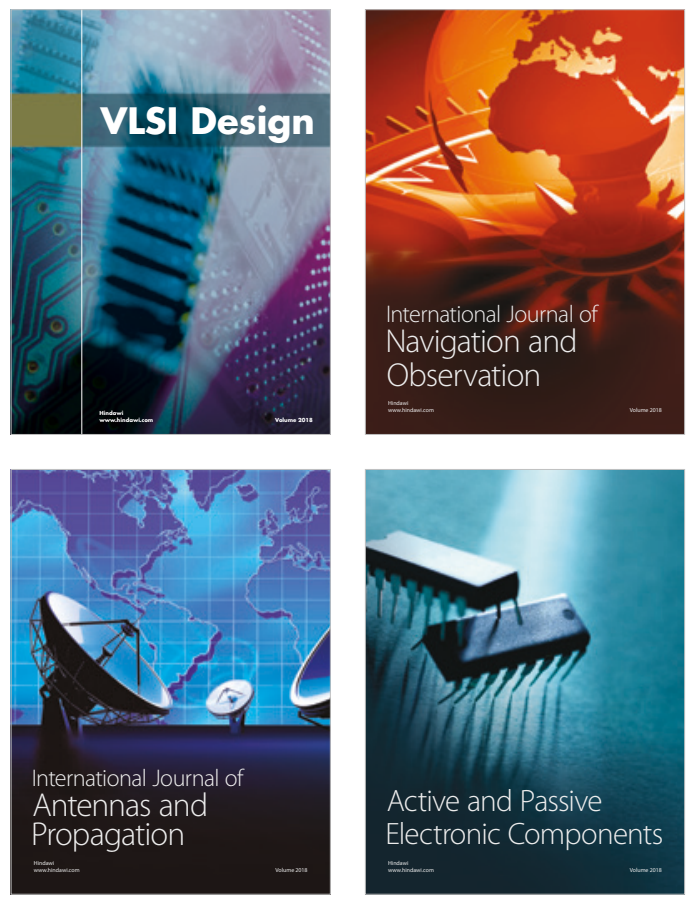
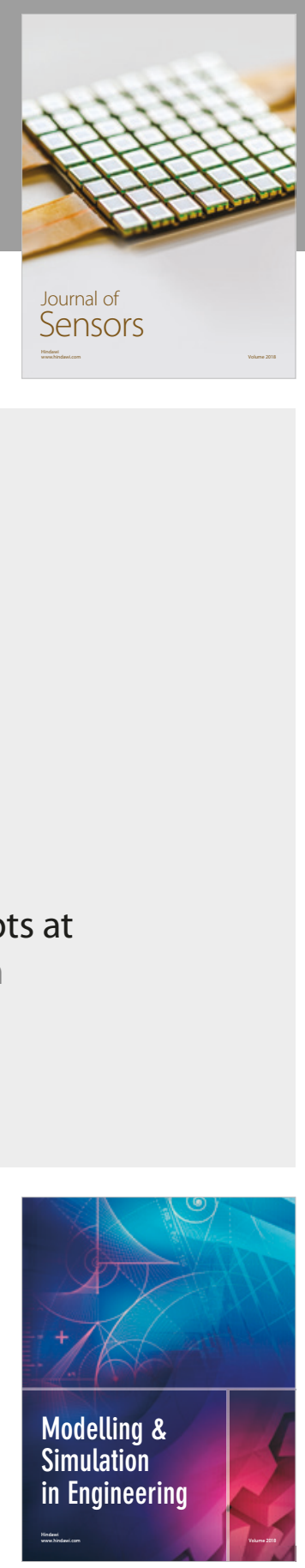

\section{Advances \\ Multimedia}
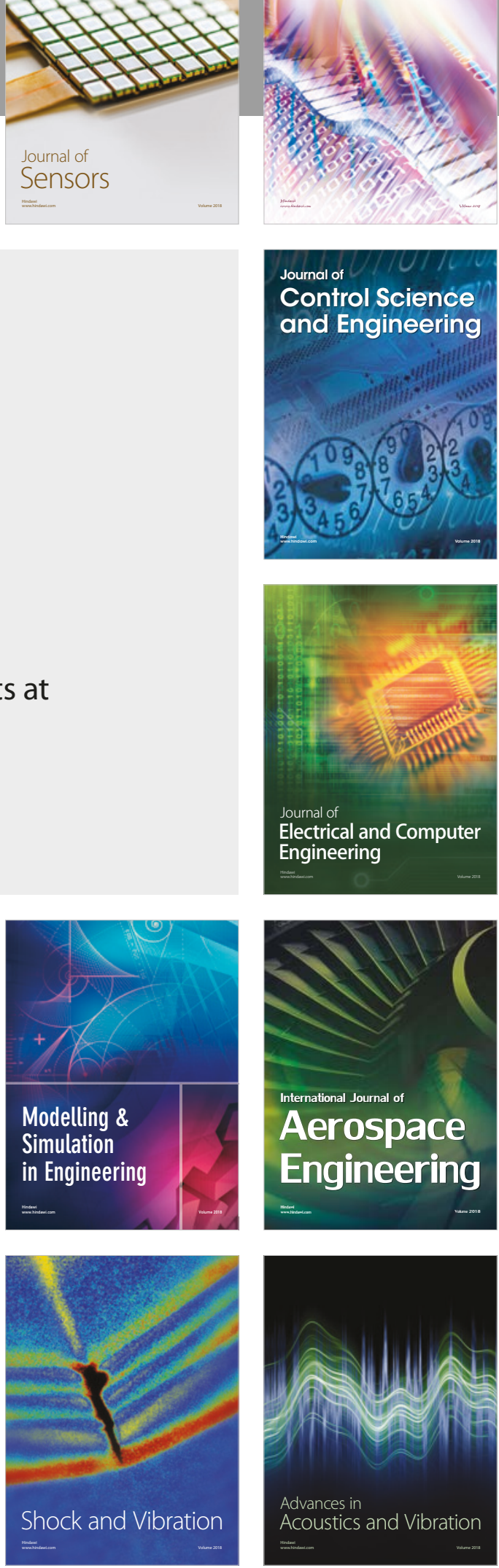